EPJ Web of Conferences 17, 11004 (2011)

DOI: $10.1051 /$ epjconf/20111711004

(C) Owned by the authors, published by EDP Sciences, 2011

\title{
Reactions with Weakly Bound Cluster Nuclei
}

\author{
Yu.E. Penionzhkevich \\ Flerov Laboratory Nuclear Reactions, JINR, 141980 Dubna, Russia
}

\begin{abstract}
Experimental excitation functions are presented for complete fusion and transfer reactions in the interaction of ${ }^{6} \mathrm{He}$ and ${ }^{6,7,9} \mathrm{Li}$ with different targets. In the case of the interaction of weakly bound nuclei the fusion process is more complex character due to the likely higher probability of the nuclei to breakup with subsequent capture of the residual nucleus (incomplete fusion). This greatly complicates the description of the interaction of such systems and leads to new unexpected effects at energies near the Coulomb barrier deeply subbarrier fusion and transfer of clusters from the weakly bound nuclei which are usually characterized by cluster structure.
\end{abstract}

Reactions involving loosely bound nuclei and occurring at energies in the vicinity of the Coulomb barrier height have many special features that have vigorously been discussed in recent years in a number of studies (see, for example, [1, 2]). These features include the enhancement of interaction cross sections in the subbarrier energy region. This effect is especially pronounced for clustering nuclei (for example, ${ }^{6,7} \mathrm{Li}$ ) [3], as well as neutron-halo nuclei (for example, ${ }^{6} \mathrm{He}$ ) [4]. Transfer, breakup, and complete fusion reactions are dominant interaction channels for such nuclei. Processes in which the breakup of loosely bound nuclei in the field of a heavy nucleus is followed by the fusion of the residual nucleus with the target nucleus have been the subject of numerous theoretical and experimental investigations. In recent years, experimental investigations along these lines have been developed quite intensively both with the aid of beams of radioactive nuclei and with the aid of beams of stable loosely bound nuclei, such as ${ }^{6,7} \mathrm{Li}$. The ${ }^{6} \mathrm{Li}$ nucleus is known have the $(\alpha+d)$-cluster structure (the energy threshold for the breakup of this nucleus into a deuteron and an alpha particle is as low as $1.47 \mathrm{MeV}$ ). The possibilities for studying reactions involving loosely bound nuclei at energies in the vicinity of the Coulomb barrier height have become substantially wider since the advent of relatively intense (up to $10^{8} \mathrm{~s}^{-1}$ ) beams of radioactive nuclei.

In the present article, we describe results of investigations performed in the past two years at the Flerov Laboratory of Nuclear Reactions at the Joint Institute for Nuclear Research (JINR, Dubna) on beams of accelerated ${ }^{6} \mathrm{He}$ and ${ }^{6,7} \mathrm{Li}$ ions. The interaction of ${ }^{6} \mathrm{Li}$ ions with ${ }^{209} \mathrm{Bi}$ target nuclei resulted in the formation of ${ }^{215} \mathrm{Rn}$ compound nuclei, whose deexcitation via neutron evaporation led to various $\mathrm{Rn}$ isotopes (in the case of the complete fusion reaction). Isotopes of $\mathrm{Po}$ and At appearing as products of reactions in which ${ }^{2} \mathrm{H}$ or ${ }^{4} \mathrm{He}$ fragments formed in ${ }^{6} \mathrm{Li}$ breakup (incomplete fusion reactions) are captured by the target nucleus $\left({ }^{209} \mathrm{Bi}\right)$. The energy dependences for the cross sections for complete fusion reaction ${ }^{209} \mathrm{Bi}\left({ }^{6} \mathrm{Li}, \mathrm{xn}\right){ }^{215-\mathrm{xn}} \mathrm{Rn}$ followed by neutron evaporation are displayed in figure 1 .

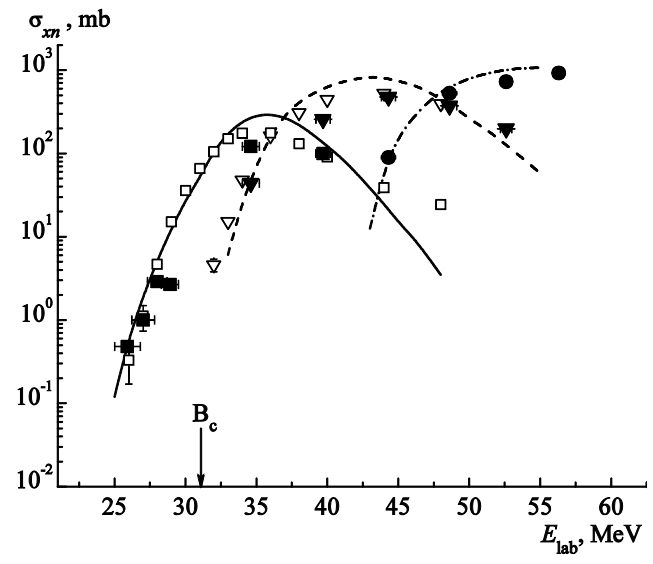

Fig. 1. Excitation functions for the reactions ${ }^{209} \mathrm{Bi}\left({ }^{6} \mathrm{Li}, \mathrm{xn}\right){ }^{215-\mathrm{xn}} \mathrm{Rn}(3 \leq \mathrm{x} \leq 5)$. The displayed points represent experimental data for the (closed and open boxes) ${ }^{209} \mathrm{Bi}\left({ }^{6} \mathrm{Li}, \mathrm{xn}\right){ }^{212} \mathrm{Rn}$, (closed and open inverted triangles) ${ }^{209} \mathrm{Bi}\left({ }^{6} \mathrm{Li}, \mathrm{xn}\right){ }^{211} \mathrm{Rn}$, and (closed circles) ${ }^{209} \mathrm{Bi}\left({ }^{6} \mathrm{Li}, \mathrm{xn}\right){ }^{210} \mathrm{Rn}$ reactions. The closed symbols stand for data of the present study, while the open boxes and open inverted triangles correspond to data from [3] for the $3 \mathrm{n}$ and $4 \mathrm{n}$ evaporation channels, respectively. The curves were calculated by using statistical model. The notation $\mathrm{B}_{\mathrm{c}}$ stands for the Coulomb barrier for ${ }^{6} \mathrm{Li}+{ }^{209} \mathrm{Bi}$ reactions.

From figure 1, one can see that, over the entire energy region considered here, the measured excitation functions agree well with the results obtained in [3]. As follows from the results of the present study, the probability of 
the fusion reaction is relatively high in the subbarrier energy region. It is noteworthy that the experimental excitation functions for evaporation reactions agree well with their counterparts calculated on the basis of the statistical model. In the case of incomplete-fusion reactions, the target nucleus captures fragments $\left({ }^{4} \mathrm{He}\right.$ or ${ }^{2} \mathrm{H}$ ) produced upon ${ }^{6} \mathrm{Li}$ breakup. The capture of product deuterons is likely to be the most probable process here. The fusion of deuterons with target nuclei in relation to the fusion of alpha particles is preferable from the point of view of the $\mathrm{Q}$ values for these reactions:

${ }^{6} \mathrm{Li}+{ }^{209} \mathrm{Bi} \rightarrow{ }^{4} \mathrm{He}+{ }^{211} \mathrm{Po}(\mathrm{Q}=+5.8 \mathrm{MeV})$,

${ }^{6} \mathrm{Li}+{ }^{209} \mathrm{Bi} \rightarrow{ }^{2} \mathrm{H}+{ }^{213} \mathrm{At} \quad(\mathrm{Q}=-10.7 \mathrm{MeV})$.

Figure 2 displays the experimentally measured excitation functions for the reactions leading to the production of the isotopes ${ }^{210} \mathrm{Po}$ and ${ }^{208} \mathrm{Po}$.

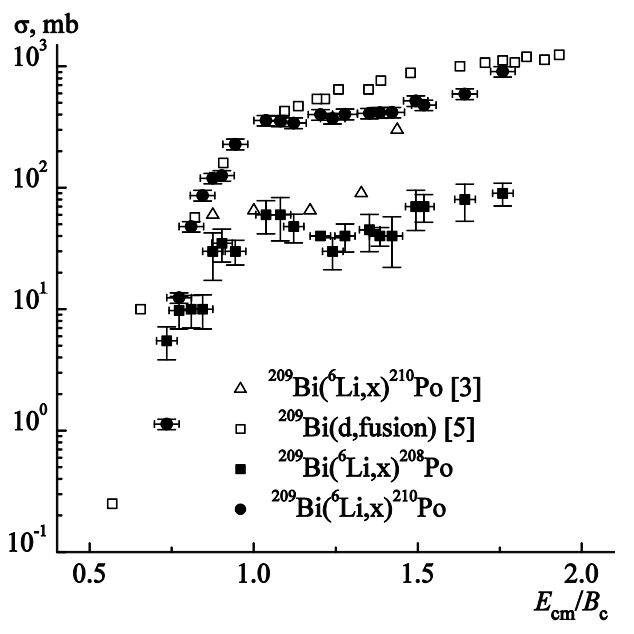

Fig. 2. Excitation functions for ${ }^{6} \mathrm{Li}+{ }^{209} \mathrm{Bi}$ reactions resulting in the production of ${ }^{208,210} \mathrm{Po}$ isotopes. The displayed closed symbols represent experimental data of the present study for the production of the isotopes (circles) ${ }^{208} \mathrm{Po}$ and (boxes) ${ }^{210} \mathrm{Po}$. The open symbols stand for experimental data on the production of the isotopes (boxes) ${ }^{208} \mathrm{Po}[5]$ and (triangles) ${ }^{210} \mathrm{Po}$ in $\mathrm{d}+{ }^{209} \mathrm{Bi}$ reactions [3].

From this figure, one can see that the cross section for the production of these isotopes amounts to a few hundred millibarns and decreases smoothly with increasing energy to a value of $10 \mathrm{mb}$ at an energy of $25 \mathrm{MeV}$. The cross section for the complete-fusion reaction at this energy value is $0.5 \mathrm{mb}$. For the sake of comparison, data on the fusion of deuterons with ${ }^{209} \mathrm{Bi}$ from [3] are also presented in figure 2. One can see that both the behavior of the excitation functions and the absolute cross-section values agree in the two cases (incomplete fusion ${ }^{6} \mathrm{Li}$ with ${ }^{209} \mathrm{Bi}$ and complete fusion of deuterons with ${ }^{209} \mathrm{Bi}$ ).

Investigations of the interaction of nuclei involving a neutron halo are of special interest. We have investigated reactions in a ${ }^{6} \mathrm{He}$ beam that result in the production of compound nuclei and their subsequent decay through the channels of neutron evaporation or fission and reactions involving neutron transfer to a target nucleus. It was indicated above that an increase in the probability of penetration (tunneling) through a potential barrier is possible for a ${ }^{6} \mathrm{He}$ nucleus because of a more extended distribution of the neutron density in it in relation to ordinary nuclei. An extended distribution of nuclear matter is characteristic of neutron-rich light nuclei, in which the presence of valence neutrons may lead to the formation of a neutron halo (neutrons cluster). In the ours experiments use was made of targets from ${ }^{206} \mathrm{~Pb}$ (in order to measure excitation functions for the fusion reactions followed by the evaporation of two neutrons from the compound nucleus, ${ }^{206} \mathrm{~Pb}\left({ }^{6} \mathrm{He}, 2 \mathrm{n}\right){ }^{210} \mathrm{Po}$ ) [4] and ${ }^{197} \mathrm{Au}$ (in order to measure excitation functions for fusion reactions followed by the evaporation of two to seven neutrons from the compound nucleus, ${ }^{6} \mathrm{He}+{ }^{197} \mathrm{Au} \rightarrow{ }^{203-\mathrm{xn}} \mathrm{Tl}$, and for reactions involving the transfer of $x$ neutrons, $\left.{ }^{6} \mathrm{He}+{ }^{197} \mathrm{Au} \rightarrow{ }^{197 \pm x n} \mathrm{Au}\right)$. Our observation of the reactions involving the evaporation of two neutrons in the subbarrier energy region and the shape of the excitation functions for these reactions give sufficient grounds to state that there is a significant enhancement of the cross sections for the fusion of ${ }^{197} \mathrm{Au}$ and ${ }^{206} \mathrm{~Pb}$ nuclei with ${ }^{6} \mathrm{He}$ nuclei near the barriers. Good agreement between the experimental excitation functions and their calculated counterparts for the reaction ${ }^{206} \mathrm{~Pb}\left({ }^{6} \mathrm{He}, 2 \mathrm{n}\right){ }^{210} \mathrm{Po}$ suggests that a sequential neutron transfer [5] for loosely bound nuclei is likely to be a dominant factor affecting the probability for the fusion of ${ }^{6} \mathrm{He}$ and ${ }^{206} \mathrm{~Pb}$ and increasing the reaction cross section in the deep-subbarrier region. The mechanism of the two-step fusion model is also supported by the fact that, as will be shown below, a large value of the cross section for the reaction involving neutron-transfer to the target nucleus from ${ }^{6} \mathrm{He}(\sigma \sim 1 \mathrm{~b})$ is observed at energies in the vicinity of the Coulomb barrier height (figure 3).

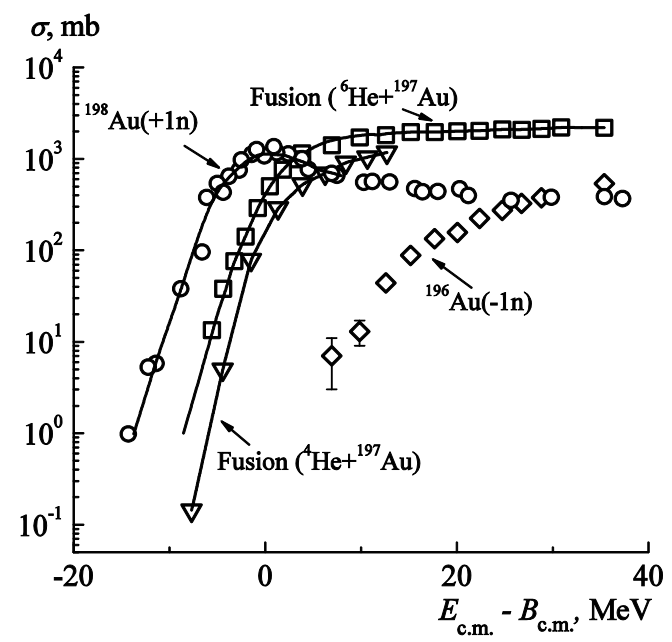

Fig. 3. Excitation functions for the total cross sections for fusion and transfer reactions induced by the interaction of ${ }^{6} \mathrm{He}$ and ${ }^{4} \mathrm{He}$ nuclei with ${ }^{197} \mathrm{Au}$ nuclei versus the difference of the projectile energy and the Coulomb barrier in the c.m. frame. The curves were drawn to guide the eye.

Such features of the interaction, which manifest themselves in the cross sections for cluster-transfer and 
complete-fusion reactions, are peculiar to many loosely bound cluster nuclei.

The reaction involving the transfer of a single neutron from ${ }^{6} \mathrm{He}$ to ${ }^{197} \mathrm{Au}$ at deep subbarrier energies $\left(\mathrm{E}_{\mathrm{c} . \mathrm{m} .}-\mathrm{B}_{\mathrm{c} . \mathrm{m} .} \leq 10 \mathrm{MeV}\right)$ has a relatively large cross section, and this may be indicative of the presence of quasifree neutrons in the $6 \mathrm{He}$ nucleus. A similar example is well known for $(d, p)$ reactions, where, in the deep-subbarier energy region, the cross section is significantly enhanced (so-called Oppenheimer-Phillips resonance [7]) because of a polarization of a loosely bound deuteron. In the case considered here, this effect can manifest itself much more strongly because of a lower binding energy of the ${ }^{6} \mathrm{He}$ nucleus in relation to the deuteron and because of a stronger force of the Coulomb repulsion of the alpha particle in the ${ }^{6} \mathrm{He}$ nucleus in relation to the proton in the deuteron. An analysis of excitation functions for transfer reactions in ${ }^{6} \mathrm{Li}$ beams corroborates the conclusion that the capture of the deuteron from ${ }^{6} \mathrm{Li}$ by the target nucleus is a dominant mechanism of such reactions at energies in the vicinity of the Coulomb barrier height. The reason for this may be that the probability of deuteron capture increases upon the excitation and polarization of the projectile nucleus in the field of the target nucleus. The results of the present study are of paramount importance for solving astrophysical problems - in particular, for obtaining deeper insight into the mechanism of the production of light elements in the Universe. In the course of nucleosynthesis, a large cross section for the interaction of loosely bound cluster nuclei $\left({ }^{6} \mathrm{He},{ }^{9} \mathrm{Li},{ }^{7} \mathrm{Be}\right)$ may change beta-decay chains leading to the formation of various elements [8]. For example, our results suggest that the reaction channels ${ }^{1} \mathrm{H}\left({ }^{6} \mathrm{He}, \mathrm{n}\right){ }^{6} \mathrm{Li},{ }^{12} \mathrm{C}\left({ }^{6} \mathrm{He}, 2 \mathrm{n}\right){ }^{16} \mathrm{O}$, ${ }^{1} \mathrm{H}\left({ }^{9} \mathrm{Li}, \mathrm{n}\right){ }^{9} \mathrm{Be},{ }^{3} \mathrm{He}\left({ }^{9} \mathrm{Li}, 2 \mathrm{n}\right){ }^{10} \mathrm{~B}$, etc., may prove to be the most probable for the synthesis of light stable nuclei.

This work was supported by the Russian Foundation for Basic Research (projects no. 09-02-91056 and 10-0200271).

\section{References}

1. I.F. Canto et al., J. Phys. G. 36, 015109 ( 2009)

2. N. Keely et al., Progress in Part. And Nucl. Phys. 59, 579 (2007)

3. M. Dasgupta et al., Phys Rev C 66, 041602R (2002).

4. Yu. Penionzhkevich et al., Phys. Rev. Lett. 96, 162701 (2006)

5. W.J. Ramler et al., Phys. Rev. C 114, 154 (1959)

6. V.I. Zagrebaev, Phys. Rev. C 67, 061601(R) (2003)

7. J.R. Oppenheimer, M. Phillips, Phys. Rev. 48, 500 (1935)

8. A. Bartlett et al., Phys. Rev. C 74, 015802 (2006) 